\title{
La Clase de Historia de la Música desde la perspectiva de los estudiantes de la Licenciatura en Música de la Universidad Autónoma de Aguascalientes, México
}

The Music History class from the perspective of Bachelor of Music degree students at Autonomous University of Aguascalientes, Mexico

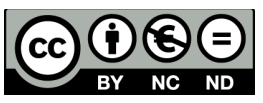

Esta obra está bajo una licencia Creative Commons Reconocimiento-No comercial-Sin Obra Derivada 


\title{
La Clase de Historia de la Música desde la perspectiva de los estudiantes de la Licenciatura en Música de la Universidad Autónoma de Aguascalientes, México
}

\author{
The Music History class from the perspective \\ of Bachelor of Music degree students \\ at Autonomous University of Aguascalientes, Mexico \\ Raúl W. Capistrán Gracia ${ }^{1}$ \\ Universidad Autónoma de Aguascalientes \\ México
}

Recibido: 23 de enero de 2020

Aprobado: 18 de mayo de 2020

\begin{abstract}
Resumen
Este artículo presenta los resultados de un estudio exploratorio de corte mixto, el cual tuvo como objetivo conocer la percepción de los estudiantes de la Licenciatura en Música de la Universidad Autónoma de Aguascalientes (México) en relación con la asignatura de Historia de la Música. A través de la técnica de la encuesta, 65 estudiantes respondieron un cuestionario que incluyó preguntas abiertas y escalas Likert. Los datos fueron capturados y analizados con los programas de computación IBM SPSS 2.0 y Atlas. ti 8.0. Los resultados reflejan aprecio hacia la asignatura, tendencia a considerarla como importante para llevar a cabo interpretaciones estilísticamente correctas y satisfacción respecto a los mecanismos de evaluación. Entre las debilidades sobresale el reclamo por procesos educativos más significativos y participativos. Se espera que los resultados contribuyan al fortalecimiento de los programas de esta materia y al diseño de planes de clase que involucren procesos educativos que impliquen mayor significancia y participación por parte de los alumnos.
\end{abstract}

Palabras clave: universidad; educación musical; musicología; docencia; enseñanza-aprendizaje

1 Doctor en Educación Musical. Profesor Investigador en la Universidad Autónoma de Aguascalientes. ORCID: 0000-0002-4594-8570. Correo: raul.capistran@edu.uaa.mx

ESCENA. Revista de las artes, 2020, Vol. 80, Núm. 1, pp. 148-178 ISSN 2215-4906 
La Clase de Historia de la Música desde la perspectiva de los estudiantes de la Artículo Licenciatura en Música de la Universidad Autónoma de Aguascalientes, México

\begin{abstract}
This article presents the results of an exploratory mixed method study, which had the goal of knowing the perception of undergraduate music major students at Autonomous University of Aguascalientes (Mexico), concerning Music History class. Through the survey technique, 65 students answered a questionnaire that included open and Likert scales questions. The data was captured and analyzed with the IBM SPSS 2.0 and Atlas.ti 8.0 computer programs. The results reflect appreciation towards the subject, a tendency to consider it as important to carry out stylistically correct interpretations and satisfaction with the evaluation mechanisms. Among the weaknesses, students claim for more meaningful and participatory educational processes. The author expects results of this study will contribute to the strengthening of subject syllabi, as well as the design of lesson plans that involve more meaningful and participatory teaching and learning processes.
\end{abstract}

Keywords: university; music education; musicology; teaching; learning process 


\section{Introducción}

La Historia de la Música² proporciona conocimientos sobre estética, estilo, morfología, organología, filosofía, sociología, entre otros, y procura el conocimiento de las historias de vida de los grandes compositores así como sus aportaciones a la música. Esa información permite que los estudiantes de las licenciaturas en música puedan entender el contexto histórico y cultural que rodeó los distintos periodos estilísticos, lo que contribuye para que tenga una comprensión más amplia y profunda del fenómeno musical; conocimiento que redunda no solo en su formación académica, intelectual y cultural, sino que también se ve reflejado en su interpretación musical (Morales Ortiz, 2012). Adicionalmente, es de todos sabido que la mejor manera de entender el presente es a través del estudio de situaciones pasadas, lo que constituye otro de los propósitos del estudio de la Historia de la Música (Carvalho-Azevedo \& Ferreira-Correa, 2014). Como afirman Casas, Escobar y Burbano (2014):

En la formación y desempeño de músicos profesionales y licenciados en Música, el saber histórico musical -respecto al conocimiento y comportamiento de los músicos y sus prácticas en la sociedad, en un determinado contexto y momentoes pertinente y necesario. (p. 380)

En México, tradicionalmente, la clase de Historia de la Música suele dividirse en tres apartados: a) el conocimiento del hecho histórico-musical; b) la audición de ejemplos musicales que ilustren la temática que se ha abordado, y c) el análisis musical panorámico (técnico, formal, armónico, estilístico...) de las obras representativas. La impartición de esta asignatura suele enfocarse en la cobertura de los contenidos del programa de materia y privilegia la construcción de saberes por medio de la cátedra expositiva y de la audición. Así, la mayoría de las veces la clase se imparte como un recuento cronológico de acontecimientos, obras musicales, características y compositores. Sin embargo, el pensamiento histórico va mucho más allá de una simple yuxtaposición de hechos cronológicos, pues la Historia debe concebirse como la unión de situaciones de vida delimitadas por el tiempo y el espacio que requieren de interpretación (Kraemer, 2000).

Más aún, desde sus orígenes, la Historia de la Música ha tenido como objetivo tratar de preservar, comprender y explicar los diversos fenómenos musicales, tanto del pasado

2 En la Licenciatura en Música de la Universidad Autónoma de Aguascalientes la clase de Historia de la Música, dentro del Plan de Estudios 2017, lleva el nombre de Música y Sociedad y se dedica al estudio de los distintos periodos estilísticos, por ejemplo, Música y Sociedad en el Barroco, Música y Sociedad en el Siglo XVIII, entre otros. En el Plan de Estudios 2009 lleva el nombre Cultura y Apreciación Musical.

ESCENA. Revista de las artes, 2020, Vol. 80, Núm. 1 (julio-diciembre), pp.148-178 
La Clase de Historia de la Música desde la perspectiva de los estudiantes de la

Artículo

Licenciatura en Música de la Universidad Autónoma de Aguascalientes, México

como del presente, así como europeos y universales. En ese esfuerzo, se ha caído en diversos vicios que van desde el análisis de objetos de distinta época y contexto cultural a través de los mismos procedimientos y bajo los mismos parámetros, hasta la exclusión de estos, por no "encajar" con las características hegemónicas europeizantes (Solorio-Farfán \& Capistrán-Gracia, 2019). En ese mismo sentido, López-Cano (2007) afirma:

El debate es etnocéntrico pues el antagonismo Musicología vs. Etnomusicología solo es posible en los sitios donde institucionalmente la investigación musical se divide en esas áreas o porque así se autodenominan las comunidades de investigación hegemónicas. Si tuviéramos que compartir nuestros departamentos universitarios con especialistas en psicología de la música o teóricos profesionales, si compartiéramos con ellos clases, estudiantes, congresos, presupuestos, sociedades científicas, etc., seguramente la discusión no se polarizaría en esta dicotomía. (p. 7)

De esta forma, los propósitos del estudio realizado, cuyos resultados se comparten en este artículo, fueron: identificar qué tan significativa es la clase de Historia de la Música para los estudiantes de la Licenciatura en Música de la Universidad Autónoma de Aguascalientes; caracterizar las acciones y recursos que, desde su perspectiva, ellos y los profesores deberían implementar para promover un proceso de enseñanza y aprendizaje más eficiente, efectivo y significativo, y conocer la opinión de los estudiantes respecto a los mecanismos de evaluación. El estudio se llevó a cabo desde una perspectiva investigativa crítica (Kincheloe \& McLaren, 2005) motivada por el propósito de que los resultados arrojen luz sobre las acciones que se deberían tomar para mejorar los procesos de enseñanza y aprendizaje de esa asignatura. Además, se tuvo en mente la posibilidad de transferir a otros espacios los hallazgos de este estudio mixto, mediante la generalización naturalista (Stake, 2005).

El artículo fue estructurado en cuatro apartados: el primero corresponde a una revisión bibliográfica que nos proporcionara un panorama sobre las investigaciones, acciones e intervenciones llevadas a cabo para hacer de la Historia de la Música una asignatura más significativa. El segundo es una exposición de los objetivos y el diseño de la investigación. El tercero abarca la descripción e interpretación de los datos recogidos. La última sección aborda las conclusiones a las que se llegó al analizar los datos a partir de la revisión bibliográfica expuesta inicialmente.

De los resultados obtenidos destaca, en primer lugar, el aprecio hacia la asignatura, seguido de una marcada tendencia a considerarla como una fuente de información importante para llevar a cabo interpretaciones musicales estilísticamente correctas y apegadas a las intenciones del compositor. Del mismo modo, fue notoria la satisfacción de los estudiantes respecto a los mecanismos de evaluación.

ESCENA. Revista de las artes, 2020, Vol. 80, Núm. 1 (julio-diciembre), pp. 148-178 
En relación con las áreas de oportunidad, sobresale el reclamo de los estudiantes por procesos de enseñanza y aprendizaje más significativos y por un involucramiento más activo en la construcción de su conocimiento, en lugar del rol pasivo que suelen desempeñar en el contexto de la cátedra expositiva. Lo anterior podría explicar por qué, para muchos de los estudiantes, la Historia de la Música no ocupa un lugar de importancia en relación con otras asignaturas. Se espera que el conocimiento de las percepciones y expectativas de los estudiantes contribuya al fortalecimiento de los programas de materia, así como al diseño de planes de clase que involucren procesos de enseñanza y aprendizaje más significativos, diversos y participativos.

\section{Revisión bibliográfica}

Para conformar este apartado, en primer lugar, se llevó a cabo una búsqueda bibliográfica sistemática, la cual tuvo como objetivo localizar fuentes que proporcionaran información sobre: investigaciones en el ámbito de la pedagogía de la Historia de la Música y propuestas metodológicas que promueven mejoras en los procesos de enseñanza-aprendizaje. La bibliografía sobre el tema es relativamente escasa, en comparación con temas tales como la educación musical, la pedagogía pianística y la teoría musical. Sin embargo, en años recientes, en el ámbito norteamericano y europeo parece haber surgido un interés por la especialización en la pedagogía de la Historia de la Música. Una búsqueda en las bases de datos de Jstor, Sage Journals, Ebsco Host y en el Open Journal System arrojó un número pequeño, pero importante, de fuentes de información, entre las que destacan los artículos publicados por Journal of Music History Pedagogy, una de las pocas revistas especializadas en ese ámbito.

Desgraciadamente en el mundo hispano la bibliografía especializada parece ser sumamente escasa. Una búsqueda en las bases de datos que incluyen artículos científicos en español, como Redalyc, Latindex, Scielo y otras similares, arrojó muy escasos resultados. Del mismo modo, se encontraron muy pocas fuentes de información en los repositorios de tesis y disertaciones.La información adicional fue obtenida de algunos libros publicados por organismos de investigación e instituciones educativas.

La metodología utilizada fue el análisis de contenido, asistido por el programa Atlas. ti 8.0. Con la ayuda de este, el investigador clasificó, estructuró, analizó la información reunida, creó un esquema general y, a partir de este, generó sus conclusiones. De las fuentes consultadas destacan los siguientes estudios y propuestas metodológicas.

ESCENA. Revista de las artes, 2020, Vol. 80, Núm. 1 (julio-diciembre), pp.148-178 
La Clase de Historia de la Música desde la perspectiva de los estudiantes de la

Artículo

Licenciatura en Música de la Universidad Autónoma de Aguascalientes, México

En el ámbito latinoamericano sobresale el estudio llevado a cabo en Colombia, por Casas, Escobar y Burbano (2014), que incluyó doce programas académicos de pregrado y Licenciatura en Música avalados por el Ministerio de Educación Nacional de ese país. La investigación tuvo como objetivo caracterizar las tendencias de formación en Historia de la Música. Los investigadores observaron dos posturas generales respecto a la importancia de esta materia. Por un lado, detectaron instituciones que consideraban esta materia como necesaria para el fomento del pensamiento crítico, la adquisición de conocimientos de la cultura musical y el desarrollo de una interpretación musical estilísticamente correcta. Por otro lado, observaron que había instituciones que desconocían las contribuciones de esta materia para la formación del músico profesional y que la limitaban a ocupar un lugar en las mallas curriculares. No en vano, Juan Carlos Montoya-Rubio afirma que:

Aunque históricamente no haya sido así, la musicología y la pedagogía de la música han de converger hacia el entendimiento. No siempre los foros musicológicos han aceptado de buen grado el papel de la pedagogía musical como parte integrante de sus postulados. Sin embargo, queda atestiguado el potencial que el conocimiento musicológico puede albergar en los desarrollos pedagógicos en el ámbito musical. (Montoya-Rubio, 2016, p. 61)

Otro estudio latinoamericano sobresaliente es el llevado a cabo en Colombia por Morales-Ortiz (2012), con el propósito de caracterizar los procesos de enseñanza de Historia de la Música en once de los programas de Licenciatura en Música de ese país. De los resultados obtenidos, cabe mencionar algunas áreas de oportunidad que son preocupantes, tales como: el desconocimiento de los docentes en temas relacionados con la pedagogía; la falta de actualización de los programas de materia; la ausencia de criterios de evaluación; el uso de una bibliografía escaza y desactualizada; el predominio del método expositivo, y el no muy alto nivel de estudios de los docentes.

En los Estados Unidos y en Europa también ha surgido un interés hacia esa asignatura y una preocupación por mejorar sus procesos de enseñanza y aprendizaje. De acuerdo con De Luca (2016), desde hace más de 15 años, los académicos de // Saggiatore musicale - Gruppo per l'Educazione Musicale, así como el Grupo de Estudio "Transmisión del Conocimiento como Objetivo Principal de la Educación Musical" del Departamento de Artes de la Universidad de Boloña han trabajado activamente en proyectos de investigación relacionados con la Educación Musical y la Historia de la Música, particularmente, han abordado el tema de lo que se debe transmitir y cómo hacerlo, con el propósito de obtener resultados significativos en la educación cultural. En ese sentido, los hallazgos parecen indicar que es vital seleccionar contenidos que sean relevantes desde el punto de vista epistemológico y estéticamente importantes, con el objetivo de promover el desarrollo de la mente, a la par que la personalidad. Adicionalmente, se debe impulsar al estudiante para que sea capaz de

ESCENA. Revista de las artes, 2020, Vol. 80, Núm. 1 (julio-diciembre), pp. 148-178 
crear redes de conocimiento que conecte con otras áreas del saber, un enfoque íntimamente conectado con la Teoría del aprendizaje significativo de Ausubel (1976, 2000).

Existe un número importante de propuestas para promover procesos de enseñanza y aprendizaje más significativos en el ámbito de la Historia de la Música. Para empezar, Burkholder (2011) considera que los profesores de esa asignatura rara vez hacen explícito que uno de los objetivos de esa disciplina es enseñar a los estudiantes a pensar como historiadores de la música. En otras palabras, los profesores deberían decodificar para los estudiantes las formas particulares de pensamiento y las habilidades disciplinarias que subyacen a la comprensión de la historia de la música para así elaborar un lenguaje común.

Por su parte, Davis (2010) destaca la importancia de desarrollar discusiones grupales sobre distintos aspectos de la clase para impulsar el pensamiento crítico y reflexivo, con base en que un involucramiento activo potencia la comprensión y la retención de la información. Sin embargo, señala también la importancia de tomar en cuenta el contexto social y profesional que rodea a los estudiantes de música. Davis, siguiendo a Nettl (1995), enfatiza la singularidad de los estudiantes de música como una comunidad cuya principal característica es la aptitud y el talento musical, pues afirma que "la aptitud musical une a este grupo y es la característica definitoria de sus miembros. Cualquier ataque a su imagen como músicos es una amenaza tanto para su identidad personal como comunitaria" (2010, p. 11). Además, Davis reconoce que en cualquier contexto escolar existe la reticencia a participar por temor a hacer el ridículo; no obstante, recalca la gran importancia que el fracaso tiene para un estudiante de música:

Antes de ofrecer una respuesta a la pregunta de un maestro o unirse a un debate, cualquier estudiante estará extremadamente consciente de cómo es percibido por sus compañeros. Estar equivocado o revelar ignorancia es una experiencia intimidante. Si bien esto es cierto en la mayoría de las situaciones de enseñanza, el problema se exacerba en el aula de historia de la música. Para el estudiante de música, va mucho más allá de parecer estúpido frente a sus compañeros. Cometer un error al responder una pregunta sobre música, podría indicar una falta de conocimiento musical, la clave para la membresía de un estudiante en la camarilla exclusiva de la escuela de música. (Davis, 2010, p. 11)

Asimismo, el autor aconseja seguir todos los pasos recomendados para impulsar una buena discusión grupal (generar preguntas abiertas, reflexivas, críticas, significativas, entre otras). Sin embargo, sugiere mantener un ambiente de máximo respeto a la diversidad de antecedentes culturales, académicos y musicales y, sobre todo, jamás poner en duda la capacidad musical del estudiante. 
La Clase de Historia de la Música desde la perspectiva de los estudiantes de la

Artículo

Licenciatura en Música de la Universidad Autónoma de Aguascalientes, México

Desgraciadamente, como se afirmó al inicio de este artículo, son muchos los profesores que se limitan a impartir sus clases a través de la cátedra expositiva y de la audición, como un mero recuento cronológico de eventos, personajes y obras. De ese modo, se hace necesario un cambio en la filosofía y en la metodología de esta asignatura. En ese sentido, en ocasión de un proyecto de intervención educativa con profesores de tiempo parcial, con el propósito de capacitarlos, mejorar sus habilidades docentes y homogeneizar el plan de estudios, Morgan-Ellis (2019) pudo constatar cómo la participación en discusiones de tipo pedagógico y el involucramiento en el desarrollo de programas de materia promovieron la confianza, la creatividad y el entusiasmo de los participantes. Morgan-Ellis concluyó que muy probablemente muchos profesores se sientan sin autoridad y estén esperando permiso para transformar sus cursos.

En lo que se refiere a la participación activa de los estudiantes, es relevante la propuesta de Cypess (2019) con respecto a la implementación del enfoque del "pensamiento histórico" de Sam Wineburg en la clase de Historia de la Música. Cypess considera que fomentar el involucramiento de los estudiantes con las fuentes primarias de información y promover una actitud investigativa podría impulsar un interés en la interpretación musical histórica, lo cual se vería reflejado en ejecuciones más informadas y correctas.

En ese mismo sentido, Kuykendall (2019) ofrece un ejercicio bastante imaginativo que puede ser de gran interés para los estudiantes: reconstruir una obra sencilla a partir de manuscritos históricos que se tengan a la mano. Kuykendall asegura que este ejercicio podría develar la complejidad que entraña una edición "limpia" e infundir un escepticismo saludable acerca de las versiones modernas.

En concordancia con Cypess (2019) y Kuykendall (2019), Erin Conor cuestiona los métodos de investigación tradicionales y propone que se debe reconsiderar "cómo introducimos a los estudiantes en la investigación dentro del contexto de la historia de la música... necesitamos ir más allá del qué y el cómo de la investigación, y comenzar a discutir el por qué" (Conor, 2019, p. 30). En ese sentido, este autor propone la utilización de bibliografía crítica, debido a que, como filosofía y como metodología, analiza los aspectos sociales y políticos que rodean una determinada información, además de problematizar el propósito y uso de esta. Además, de acuerdo con Conor, este enfoque fomenta el pensamiento crítico de los estudiantes para actuar sobre ese conocimiento y constituye una metodología que promueve la lectura de fuentes de información de una manera reflexiva y analítica, al mismo tiempo que permite reducir el típico "escaneo" que muchos estudiantes llevan a cabo, con el propósito de evitar el desagrado de leer a profundidad (Cochran, 2019).

ESCENA. Revista de las artes, 2020, Vol. 80, Núm. 1 (julio-diciembre), pp. 148-178 
Por su parte, Lowe (2010) propone concentrarse en hacer que la historia de la música sea tangible y relevante en la vida de los estudiantes. Por ello, proporciona tres ejemplos de actividades de clase relativas a la música del siglo XVIII, para lograr ese contacto entre el "entonces" y el "ahora". Sin embargo, Lowe considera que para tener el tiempo necesario para conectar la historia de la música europea con las experiencias de la vida cotidiana de los estudiantes, es necesario romper con uno de los paradigmas en la impartición de esta asignatura, el cual corresponde al objetivo poco realista de dar un panorama histórico-musical lo más completo posible.

Una propuesta interesante es la de Stimeling y Katz (2012), quienes sugieren que los estudiantes deberían involucrarse en ejercicios de composición y creación de canciones, para luego reflexionar y analizar lo que la música puede significar a través del tiempo y la configuración social. En esa misma línea, Yang (2012) propone implicarse en la interpretación de algunas obras representativas, en lugar de limitarse a la escucha de grabaciones, como una herramienta para "vivir" la Historia de la Música y darle un mayor significado. Empero, Yang advierte que se debe ser cuidadoso con esta estrategia, ya que la interpretación no es el objetivo principal del curso.

En ese mismo sentido, Knyt (2013) sugiere darle un carácter más pragmático al trabajo escrito que suele formar parte de la evaluación final. Adicionalmente recomienda darle el formato de "notas al programa", de tal suerte que el estudiante sienta que combina la teoría con la práctica. Algo similar propone Haefeli (2013) al recomendar el formato electrónico del blog como medio para publicar los trabajos escritos finales.

Como cualquier otra disciplina académica, la didáctica de la Historia de la Música puede y debe enriquecerse a través de la investigación empírica. Actualmente, la bibliografía que ha resultado de reportes de investigación es aún escasa, sin embargo, los hallazgos son importantes. La información sobre el impacto que los antecedentes de los estudiantes ejercen en su actitud y desempeño académico y la efectividad de ciertas estrategias para mejorar los procesos de enseñanza y aprendizaje pueden ser de gran valía. Del mismo modo, los resultados de estos estudios pueden ayudar a los maestros de Historia de la Música a estructurar sus cursos de una manera más informada y fundamentada, y darles ideas de cómo impartir mejor sus clases. En relación con esto, Dirkse sostiene que:

Los resultados de estos estudios pueden enseñarnos más sobre nuestra población estudiantil y ofrecer ideas para mejorar nuestros métodos de enseñanza. Más importante aún, este pequeño cuerpo de literatura puede estimular ideas para ir más allá con respecto a estas áreas de investigación. (Dirkse, 2011, p. 26) 
La Clase de Historia de la Música desde la perspectiva de los estudiantes de la

Artículo

Licenciatura en Música de la Universidad Autónoma de Aguascalientes, México

\section{El diseño de la investigación}

Esta investigación parte del objetivo general de conocer qué tan significativa es la clase de Historia de la Música desde la perspectiva de los estudiantes de la Licenciatura en Música de la Universidad Autónoma de Aguascalientes. Para ello, se plantearon los siguientes objetivos específicos: determinar el grado de identificación de los estudiantes con respecto a la asignatura de Historia de la Música; conocer la percepción que los estudiantes tienen acerca del valor que esta asignatura tiene en su formación profesional; determinar los aspectos de la Historia de la Música considerados como más importantes por los estudiantes; caracterizar las acciones y recursos que, desde la perspectiva de los estudiantes, los profesores deberían utilizar para promover un proceso de enseñanza y aprendizaje más eficiente, efectivo y significativo; caracterizar las acciones y recursos que, desde su propia perspectiva, los estudiantes deberían llevar a cabo para coadyuvar a un proceso de enseñanza y aprendizaje más eficiente, efectivo y significativo, y caracterizar la opinión de los estudiantes con respecto a los mecanismos de evaluación.

En cuanto a los métodos y materiales, la investigación fue de tipo exploratorio y de corte mixto. Para la recopilación de información se diseñó e implementó un cuestionario constituido por preguntas contextuales, con escalas tipo Likert. Con el propósito de evitar los sesgos, en la medida de lo posible, muchas de las preguntas tipo Likert incluyeron la opción otro, de tal manera que el encuestado pudiera expresar cualquier otra opinión no considerada en las opciones. Del mismo modo, el cuestionario incluyó preguntas con respuestas múltiples. Para validar la escala se utilizó la técnica de juicio de expertos, en la que participaron cinco profesores especializados en la impartición de la asignatura. Para ese propósito se elaboró un cuestionario para determinar la suficiencia, claridad, coherencia y relevancia de cada uno de los ítems y se implementó el procedimiento recomendado por Escobar-Pérez y Cuervo-Martínez (2008). Sin embargo, con el propósito de determinar la validez de contenido y la concordancia entre los jueces, se utilizó el análisis estadístico de Hernández Nieto, que arrojó un coeficiente de 0.874. De acuerdo con el autor del cálculo estadístico, un coeficiente entre 0.80 y 0.90 puede categorizarse como "bueno". Además, para determinar el nivel de confiabilidad de la escala, se requirió el estadístico Alfa de Cronbach, con el que se obtuvo un coeficiente de $a=.984$.

Para propósito del estudio se tomó una muestra por conveniencia estadísticamente representativa, conformada por 65 de los 76 estudiantes inscritos en la clase de Historia de la Música, del Departamento de Música de la Universidad Autónoma de Aguascalientes, México (UAA). Esta muestra tuvo un margen de error del $5 \%$ y un nivel de confianza del 95\%. Las edades de los 65 participantes se encontraban en un rango de 19 a 29 años, con una desviación estándar de 2.7 años; 35 fueron hombres y 30 fueron mujeres (Tabla 1).

ESCENA. Revista de las artes, 2020, Vol. 80, Núm. 1 (julio-diciembre), pp. 148-178 
Tabla 1. Cantidad de estudiantes por materia

\begin{tabular}{|l|c|c|}
\hline \multicolumn{1}{|c|}{ Nombre de la Asignatura } & $\begin{array}{c}\text { Número de estudiantes } \\
\text { inscritos }\end{array}$ & $\begin{array}{c}\text { Número de estudiantes } \\
\text { encuestados }\end{array}$ \\
\hline Música y Sociedad Medieval y Renacentista & 24 & 20 \\
\hline Música y Sociedad en el Siglo XVIII & 25 & 22 \\
\hline Cultura y Apreciación Musicales VI & 17 & 14 \\
\hline Cultura y Apreciación Musicales VIII & 10 & 9 \\
\hline Total & 76 & 65 \\
\hline
\end{tabular}

Fuente: Tabla creada por el autor con la información derivada de la investigación.

Tabla 2. Número de estudiantes por instrumento musical

\begin{tabular}{|l|c|l|c|l|c|}
\hline Instrumento & Frecuencia & Instrumento & Frecuencia & Instrumento & Frecuencia \\
\hline Violín & 9 & Flauta & 1 & Contrabajo & 1 \\
\hline Viola & 4 & Oboe & 1 & Trompeta & 2 \\
\hline Cello & 6 & Clarinete & 1 & Trombón & 2 \\
\hline Piano & 11 & Percusiones & 2 & Canto & 7 \\
\hline Guitarra & 18 & & & & \\
\hline
\end{tabular}

Fuente: Tabla creada por el autor con la información derivada de la investigación.

\section{El procedimiento de la investigación}

Los estudiantes de la Licenciatura en Música de la UAA fueron encuestados los días 5, 6 y 7 de junio de 2019, es decir, al final del semestre. El cuestionario fue aplicado por el propio investigador. El llenado de los cuestionarios fue voluntario y anónimo. Los datos cuantitativos fueron capturados, procesados y analizados con la ayuda del programa Statistics Package for the Social Sciences IBM SPSS 2.0. Para llevar a cabo el análisis de los datos obtenidos a través de las respuestas proporcionadas en las preguntas abiertas, se utilizó la metodología propuesta por González (1999) en su capítulo de libro "La sistematización y el análisis de los datos cualitativos", la cual involucró los siguientes cinco pasos: 
La Clase de Historia de la Música desde la perspectiva de los estudiantes de la

Artículo

Licenciatura en Música de la Universidad Autónoma de Aguascalientes, México

Primero, se realizó una conceptualización que consistió en ordenar la información recabada en unidades de análisis. En otras palabras, se identificaron los diversos pensamientos e ideas que el participante proporcionó. Luego, se llevó a cabo la categorización: después de identificar las unidades de análisis, los investigadores las categorizaron, es decir, congregaron los pensamientos o ideas en grupos que las contuvieran. Este paso incluyó la identificación de subcategorías, es decir, subgrupos de unidades de análisis. En tercer lugar, se procedió a la organización, esta etapa implicó encontrar la lógica que conectara las unidades de análisis con las subcategorías, las subcategorías con las categorías y las categorías entre sí. Se trató de un proceso que tuvo como propósito visualizar los niveles de importancia de las categorías, su interrelación y la manera en la que conforman un todo. El cuarto paso, y último del proceso de inducción, correspondió a una estructuración en la que las partes del todo se ordenaron y se graficaron en un mapa conceptual. Finalmente, se efectuó el análisis y la interpretación de los datos, esta fase de la investigación implicó partir del mapa conceptual creado e identificar las categorías y subcategorías de cada nivel que presentaban el mayor número de unidades de análisis, con el objetivo de identificar los patrones de significación. En estos procesos (conceptualización, categorización, organización, estructuración y análisis e interpretación de los datos), se utilizó el programa Atlas.ti.

\section{Los resultados de la investigación}

La primera pregunta del cuestionario tuvo como objetivo conocer las expectativas profesionales de los estudiantes y fue redactada como: ¿Cuál es tu principal expectativa cuando termines la licenciatura en música? La opción "ser maestro de mi instrumento" ocupó el primer lugar, con 27 frecuencias (22.3\%), seguida de "ser instrumentista y tocar en grupos de cámara", con 26 frecuencias (21.5\%). Las respuestas reflejan los ámbitos en los que enfatiza el plan de estudios de la Licenciatura en Música: la ejecución y la educación.

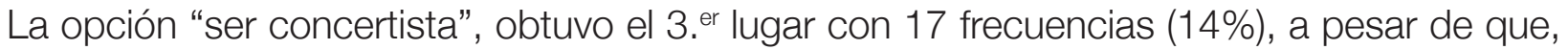
en general, los maestros del Departamento de Música hemos tratado de ampliar la visión de nuestros estudiantes respecto a las opciones laborales más viables en la entidad. La respuesta "ser atrilista de una orquesta o banda" alcanzó 13 frecuencias (10.7\%), algo que sorprende, dado que representa una de las opciones laborales de la entidad y que es enfatizada en la carrera a través de la materia de Prácticas Orquestales. La respuesta "ser maestro de materias teóricas" recibió el mismo número de frecuencias. La opción "ser maestro de educación musical”, quedó en un preocupante 5. lugar con solo nueve frecuencias (7.4\%), a pesar de que la docencia en el nivel educativo básico representa una importante fuente de ingresos para los músicos profesiones. En cuanto a la opción "ser maestro de Historia de la Música", esta obtuvo solo cuatro frecuencias (3.3\%), a pesar de que suele representar la manera más común en que muchos maestros de nivel medio superior y superior completan su carga académica. Esta pregunta incluyó la opción de respuesta "otros", a fin de permitir

ESCENA. Revista de las artes, 2020, Vol. 80, Núm. 1 (julio-diciembre), pp. 148-178 
a los estudiantes incluir cualquier otra expectativa no considerada por el investigador. "Ser compositor", "Ser director de orquesta" y "Realizar proyectos interdisciplinares de música nueva" fueron tres expectativas adicionales con cuatro (3.3\%), $2(1.7 \%)$ y una $(0.8 \%)$ frecuencias, respectivamente (Tabla 3). Cabe mencionar que, en esta pregunta, los estudiantes podían marcar tantas opciones como así lo consideraran.

Tabla 3. Expectativas de los estudiantes

\begin{tabular}{|l|c|c|c|}
\hline \multicolumn{1}{|c|}{ Expectativa } & \multicolumn{2}{c|}{ Respuestas } \\
\cline { 2 - 4 } & Frecuencias & Porcentaje & $\begin{array}{c}\text { Porcentaje } \\
\text { de casos }\end{array}$ \\
\hline Ser maestro de instrumento & 27 & $22.3 \%$ & $42.9 \%$ \\
\hline Ser instrumentista y tocar en grupos de cámara & 26 & $21.5 \%$ & $41.3 \%$ \\
\hline Ser concertista & 17 & $14.0 \%$ & $27.0 \%$ \\
\hline Ser atrilista de una orquesta o banda & 13 & $10.7 \%$ & $20.6 \%$ \\
\hline Ser maestro de materias teóricas & 13 & $10.7 \%$ & $20.6 \%$ \\
\hline Ser maestro de educación musical & 9 & $7.4 \%$ & $14.3 \%$ \\
\hline Ser compositor & 9 & $7.4 \%$ & $14.3 \%$ \\
\hline Ser maestro de Historia de la Música & 4 & $3.3 \%$ & $6.3 \%$ \\
\hline Ser director de orquesta & 2 & $1.7 \%$ & $3.2 \%$ \\
\hline $\begin{array}{l}\text { Realizar proyectos interdisciplinares } \\
\text { de música nueva }\end{array}$ & 1 & $0.8 \%$ & $1.6 \%$ \\
\hline
\end{tabular}

Fuente: Tabla creada por el autor con la información derivada de la investigación.

Uno de los "misterios" de la clase de Historia de la Música es conocer el grado de identificación de los estudiantes con esta materia; es decir, conocer si los estudiantes gustan de esta asignatura o si solamente la toman porque es un requisito para cumplir con el plan de estudios y poder obtener un título de licenciatura. Así, el cuestionario incluyó la pregunta "¿Qué tanto te gusta la clase de historia de la música?”. En su gran mayoría, los estudiantes respondieron gustar de la materia: 38.5\% afirmó gustar "mucho" de esa asignatura, 35.4\% consideró hacerlo "bastante" y 23.1\% respondió "algo" (Figura 1). 
La Clase de Historia de la Música desde la perspectiva de los estudiantes de la

Figura 1. Grado de gusto por la asignatura

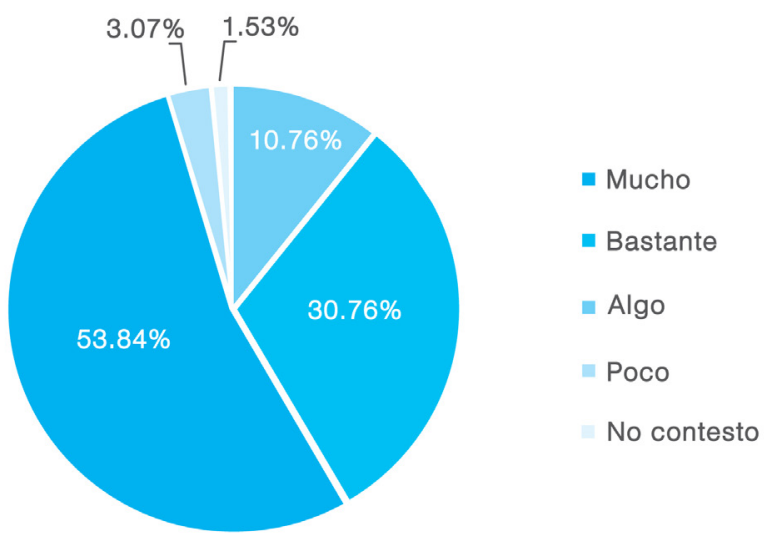

Fuente: Creación del autor con los datos derivados de la investigación.

Figura 2. Grado de utilidad de la asignatura desde la perspectiva de los estudiantes.

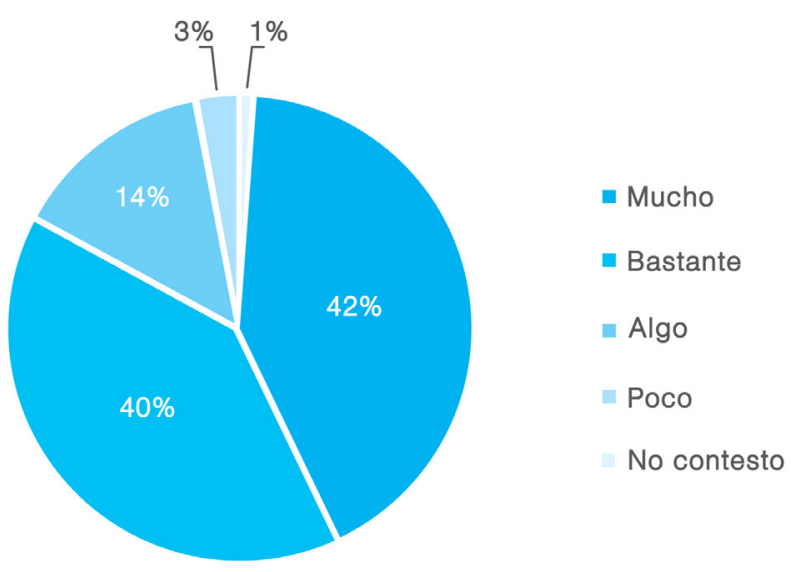

Fuente: Creación del autor con los datos derivados de la investigación. tudiantes le dan a esta asignatura, pues 35.4\% de los estudiantes eligió "Aprender sobre el desarrollo de los estilos, las formas y los lenguajes musicales", 20\% seleccionó "Conocer el contexto filosófico, histórico, cultural, social, artístico que rodeó un periodo estilístico" (un aspecto íntimamente relacionado con la adquisición de una cultura general), 18.5\% 
indicó "Adquirir algunas herramientas para analizar la música", y un 9.2\% marcó "Conocer las obras más importantes del mundo de la música occidental y sus características". Esta pregunta también incluyó la opción de respuesta "otro".(Tabla 4). Un aspecto interesante a destacar de las respuestas proporcionadas fue que la vida y la obra de los grandes compositores (dos de los aspectos en los que se suelen enfatizar en la clase de Historia de la Música) obtuvieron frecuencias muy bajas.

Tabla 4. Aspectos de la clase que, desde la perspectiva de los estudiantes, se deberían enfatizar más

\begin{tabular}{|l|c|c|}
\hline \multicolumn{1}{|c|}{ Opción de respuesta } & Frecuencia & Porcentaje \\
\hline $\begin{array}{l}\text { Aprender sobre el desarrollo de los estilos, las formas y los } \\
\text { lenguajes musicales }\end{array}$ & 23 & 35.4 \\
\hline $\begin{array}{l}\text { Conocer el contexto filosófico, histórico, cultural, social } \\
\text { y artístico que rodeó un periodo estilístico }\end{array}$ & 13 & 20.0 \\
\hline Adquirir algunas herramientas para analizar la música & 12 & 18.5 \\
\hline $\begin{array}{l}\text { Conocer las obras más importantes del mundo de la música } \\
\text { Occidental y sus características }\end{array}$ & 6 & 9.2 \\
\hline Aprender aspectos relacionados con la vida de los compositores & 3 & 4.6 \\
\hline Otro: Todos los aspectos son importantes & 2 & 3.1 \\
\hline Otro: Vida de compositores de Guitarra & 1 & 1.5 \\
\hline $\begin{array}{l}\text { Otro: Conocer música de los instrumentos que tocamos } \\
\text { los estudiantes }\end{array}$ & 1 & 1.5 \\
\hline Perdidos & & 6.2 \\
\hline
\end{tabular}

Fuente: Tabla creada por el autor con la información derivada de la investigación

Con el propósito de conocer con mayor profundidad la percepción de los estudiantes respecto a los aspectos más significativos de la asignatura, se incluyó la pregunta abierta "¿Por qué consideras que tu maestro debería enfatizar más ese aspecto de la clase?”. Después

3 La categoría "perdidos" se refiere a aquellas respuestas proporcionadas por los estudiantes que no guardan relación alguna con la pregunta. Por ejemplo, la respuesta: "Creo que la clase es muy aburrida", aunque arroja información importante, no corresponde a la pregunta ni arroja información sobre la variable.

ESCENA. Revista de las artes, 2020, Vol. 80, Núm. 1 (julio-diciembre), pp.148-178 
La Clase de Historia de la Música desde la perspectiva de los estudiantes de la

Artículo

Licenciatura en Música de la Universidad Autónoma de Aguascalientes, México

de llevar a cabo el análisis para las respuestas abiertas, descrito anteriormente, se determinaron tres grandes categorías:

- Aspectos que deben enfatizarse porque contribuyen a realizar una mejor interpretación musical.

- Aspectos que deben enfatizarse porque contribuyen a una cultura musical general.

- Aspectos que deben enfatizarse porque no son comprensibles para los estudiantes.

En la primera categoría, el $18.46 \%$ de los estudiantes consideró que el estudio de los estilos, los lenguajes y las formas musicales contribuía a realizar una mejor interpretación musical, mientras que $12.31 \%$ afirmó que la adquisición de algunas herramientas para el análisis de las obras también contribuía positivamente en la interpretación. En ese mismo sentido, 3.08\% pensó que todos los aspectos de la clase de Historia de la Música debían enfatizarse, ya que todo ese conocimiento redundaba en una mejor interpretación (Tabla 5). Los estudiantes dieron respuestas como:

Opción: Aprender aspectos relacionados con la vida de los compositores.

¿Por qué?: Porque conocer las vivencias del compositor nos ayuda a comprender qué sentía al momento de escribir una obra y así poder dar una mejor interpretación.

Opción: Conocer el contexto filosófico, histórico, cultural, social, artístico que rodeó un periodo estilístico.

¿Por qué?: Para saber más sobre la obra, conocer su pasado, como se tocaba y en qué condiciones, y así dar una buena presentación.

Opción: Aprender aspectos relacionados con la vida de los compositores.

¿Por qué?: En mi repertorio personal como músico, a la hora que hago una interpretación, me gustaría saber qué pasaba por la cabeza del compositor y sus aspectos sociales, etcétera.

Opción: Adquirir algunas herramientas para analizar la música.

¿Por qué?: Porque pienso que si se relaciona este aspecto con el contexto social y los demás temas que se abarcan puede darnos más herramientas para comprender e interpretar de mejor manera las piezas de ese periodo.

Opción: Aprender sobre el desarrollo de los estilos, las formas, los lenguajes musicales. ¿Por qué?: Porque al conocer cómo fue hecha una obra, puedo entender el objetivo que buscaba lograr el compositor, y así reproducirlo al interpretar una composición.

ESCENA. Revista de las artes, 2020, Vol. 80, Núm. 1 (julio-diciembre), pp. 148-178 
Tabla 5. Aspectos que deben enfatizarse porque contribuyen para realizar una mejor interpretación

\begin{tabular}{|l|c|c|}
\hline \multicolumn{1}{|c|}{ Opción de respuesta } & Frecuencia & Porcentaje \\
\hline $\begin{array}{l}\text { Aprender sobre el desarrollo de los estilos, las formas } \\
\text { y los lenguajes musicales }\end{array}$ & 12 & $18.46 \%$ \\
\hline $\begin{array}{l}\text { Conocer el contexto filosófico, histórico, cultural, social, artístico } \\
\text { que rodeó un periodo estilístico }\end{array}$ & 3 & $4.62 \%$ \\
\hline Adquirir algunas herramientas para analizar la música & 8 & $12.31 \%$ \\
\hline $\begin{array}{l}\text { Conocer las obras más importantes del mundo de la música } \\
\text { occidental y sus características }\end{array}$ & 3 & $4.62 \%$ \\
\hline Todo & 2 & $3.08 \%$ \\
\hline Aprender aspectos relacionados con la vida de los compositores & 2 & $3.08 \%$ \\
\hline Total & 30 & $46.17 \%$ \\
\hline
\end{tabular}

Fuente: Tabla creada por el autor con la información derivada de la investigación.

En la segunda categoría, 10.77\% estudiantes consideraron que aprender sobre el desarrollo de los estilos, las formas y los lenguajes musicales debía enfatizarse porque contribuía a su cultura general. se igual manera, 9.23\% afirmó que conocer el contexto filosófico, histórico, cultural, social, artístico que rodeó un periodo estilístico ayudaba al mismo propósito. Con menores porcentajes (6.15\% y 4.62\%), se encontró que adquirir algunas herramientas para analizar la música y conocer las obras más importantes del mundo de la música occidental y sus características fomentaban el desarrollo de una cultura general (Tabla 6).

Tabla 6. Aspectos que deben enfatizarse porque contribuyen al desarrollo de una cultura general

\begin{tabular}{|l|c|c|}
\hline \multicolumn{1}{|c|}{ Opción de respuesta } & Frecuencia & Porcentaje \\
\hline $\begin{array}{l}\text { Aprender sobre el desarrollo de los estilos, las formas } \\
\text { y los lenguajes musicales. }\end{array}$ & 7 & $10.77 \%$ \\
\hline $\begin{array}{l}\text { Conocer el contexto filosófico, histórico, cultural, social, artístico } \\
\text { que rodeó un periodo estilístico }\end{array}$ & 6 & $9.23 \%$ \\
\hline Adquirir algunas herramientas para analizar la música & 4 & $6.15 \%$ \\
\hline $\begin{array}{l}\text { Conocer las obras más importantes del mundo de la música } \\
\text { occidental y sus características. }\end{array}$ & 3 & $4.62 \%$ \\
\hline Conocer más música de nuestro instrumento. & 1 & $1.54 \%$ \\
\hline Aprender aspectos relacionados con la vida de los compositores & 1 & $1.54 \%$ \\
\hline
\end{tabular}

Fuente: Tabla creada por el autor con la información derivada de la investigación.

ESCENA. Revista de las artes, 2020, Vol. 80, Núm. 1 (julio-diciembre), pp.148-178 
La Clase de Historia de la Música desde la perspectiva de los estudiantes de la

Algunas de las respuestas que se consideraron dentro de esta categoría fueron:

Opción: Aprender sobre el desarrollo de los estilos, las formas y los lenguajes musicales.

¿Por qué?: Porque, como músicos, es importante entender cómo funciona la música y saber cómo distinguir un estilo de otro.

Opción: Conocer el contexto filosófico, histórico, cultural, social, artístico que rodeó un periodo estilístico.

¿Por qué?: Me parece importante, ya que en el mundo profesional no solo basta con saber tocar.

Opción: Aprender sobre el desarrollo de los estilos, las formas, los lenguajes musicales.

¿Por qué?: Al final, lo que diferencia a un periodo de otro o a un estilo de otro son los cambios que se implementaron en la forma de componer la música; los cambios en el lenguaje musical, etcétera".

Opción: Conocer las obras más importantes de un compositor y sus características. ¿Por qué?: Porque creo que es muy enriquecedor conocer y comparar los estilos de los músicos más destacados de la historia y aprender a analizar la música.

Por lo que respecta a la categoría "Aspectos que deben enfatizarse porque no son comprensibles para los estudiantes", se obtuvieron los siguientes resultados mostrados en la tabla 7.

Tabla 7. Aspectos que deben enfatizarse porque no son comprensibles para los estudiantes

\begin{tabular}{|l|c|c|}
\hline \multicolumn{1}{|c|}{ Aspecto } & Frecuencia & Porcentaje \\
\hline $\begin{array}{l}\text { Aprender sobre el desarrollo de los estilos, las formas } \\
\text { y los lenguajes musicales }\end{array}$ & 2 & $3.08 \%$ \\
\hline $\begin{array}{l}\text { Conocer el contexto filosófico, histórico, cultural, social, artístico } \\
\text { que rodeó un periodo estilístico }\end{array}$ & 3 & $4.62 \%$ \\
\hline Total & 5 & $7.70 \%$ \\
\hline
\end{tabular}

Fuente: Tabla creada por el autor con la información derivada de la investigación 
Algunas de las respuestas consideradas dentro de esta categoría fueron:

Opción: Conocer el contexto filosófico, histórico, cultural, social, artístico que rodeó un periodo estilístico.

¿Por qué?: Porque a veces no me queda claro (en lo personal) qué quiso decir o explicar.

Opción: Adquirir herramientas para analizar la música.

¿Por qué?: Personalmente batallo un poco en el análisis y sé que es muy importante y lo va a ser más a futuro, que espero tocar más obras complejas para entenderlas mejor.

Finalmente, hubo ocho ítems (12.31\%) que se clasificaron como "perdidos", debido a dos razones: el estudiante no había proporcionado respuesta a alguna de las dos preguntas - por lo que no era posible relacionarlas - o la respuesta no correspondía a la pregunta.

Desde hace cuatro años, el Cuerpo Académico "Educación y Conocimiento de la Música" implementó la participación de los estudiantes en el programa de radio titulado "Ventana al Sonido", como una de las actividades de aprendizaje de la materia. Esta consiste en la elaboración de un guion de radio sobre un tema histórico-musical específico, para compartirlo con los radioescuchas de nuestra estación universitaria. Con la intensión de evaluar esta actividad de aprendizaje desde la perspectiva de los estudiantes, se incluyó la pregunta abierta "¿Qué opinas de la producción del programa de Radio «Ventana al Sonido" como parte de tu formación académica?" (Tabla 8). El análisis de la información arrojó seis categorías:

a) Estrategia para el aprendizaje de contenidos curriculares. Las respuestas aludían a la actividad como un recurso que impulsaba a la investigación y fomentaba el aprendizaje de información relativa a un tema. Entre esas respuestas están las siguientes: "Opino que nos ayuda, porque estudiamos más a fondo un tema y aprendemos a explicarlo mejor" o "me parece una excelente idea, ya que conocemos a fondo nuestro tema". Este tipo de respuestas alcanzó un 29.23\% e indican que la actividad cumple con su propósito principal.

b) Propósito trascendente de la actividad. En esta categoría se incluyeron respuestas que aludían a la trascendencia cultural y social de difundir la información aprendida en clase, lo que hacía que la actividad tuviera algún sentido o propósito para los estudiantes, por ejemplo: "Es bueno porque permite dar a conocer muchos datos de la historia de la música a personas que no se dedican a esto" y "nos ayuda a difundir la información a más gente. Es importante compartir la historia, ya que normalmente la gente que no es músico no sabe de historia". Las respuestas en esta categoría sumaron 24.62\%.

ESCENA. Revista de las artes, 2020, Vol. 80, Núm. 1 (julio-diciembre), pp.148-178 
La Clase de Historia de la Música desde la perspectiva de los estudiantes de la

Artículo

Licenciatura en Música de la Universidad Autónoma de Aguascalientes, México

c) Agrado/desagrado. Las respuestas listadas en esta categoría mencionaban el agrado o desagrado hacia la actividad, sin dar explicación alguna. Un par de ellas son: "Me encanta que nos den la oportunidad de participar" o "no me gusta". Solo $7.69 \%$ de los participantes opinaron que la actividad les desagradaba, mientras que $13.85 \%$ opinó que les agradaba llevarla a cabo.

d) Recurso para la formación como futuro profesional de la música. Las respuestas enfatizaban en la importancia de involucrarse en la actividad, como medio para adquirir herramientas de utilidad para su vida futura profesional. Algunas respuestas fueron: "Nos da experiencia frente al micrófono como divulgadores musicales", "nos da opciones laborales" y "nos acerca a los medios de comunicación, saber cómo trabajar en ellos". En esta categoría las respuestas sumaron 20\%.

e) Logística y organización. Esta categoría consistente en opiniones sobre esos aspectos. Estas respuestas proporcionaron información importante a los integrantes del cuerpo académico para mejorar la producción y difusión del programa de radio. Algunas opiniones fueron: "Lo que no me gusta es que hay tantos programas que luego ni sabemos cuándo es" y "creo que en ocasiones está muy forzada la entrega [del guion] y lo hace más por entregar que por aportar a la radio. Sería mejor seleccionar a quienes se motivan por grabar en cada grupo y hacer programas de calidad". Solo 10 participantes opinaron al respecto sumando $15.38 \%$.

f) Falta de sentido de la actividad. Las respuestas aludían a la falta de relevancia o pertinencia de la actividad. Aunque solo $10.77 \%$ de los estudiantes dieron estas opiniones, ellas representan una llamada de atención, pues implican que el estudiante desconoce la finalidad de esta actividad. Entre las opiniones, se encontraron las siguientes: "No entiendo mucho su función dentro de la materia"; "no creo que sea relevante", y "realmente no lo considero necesario, ya que podríamos realizar alguna otra actividad con mayor impacto y sin necesidad de trasladarse" [a la estación de radio]. 
Tabla 8. Resumen por categorías de la opinión sobre la producción del programa de Radio "Ventana al Sonido".

\begin{tabular}{|c|c|c|c|}
\hline \multicolumn{2}{|c|}{ Categoría } & Frecuencia & Porcentaje \\
\hline \multicolumn{2}{|c|}{ Estrategia para el aprendizaje de contenidos curriculares } & 19 & 29.23 \\
\hline \multicolumn{2}{|c|}{ Propósito trascendente de la actividad } & 16 & 24.62 \\
\hline \multirow{2}{*}{ Agrado/desagrado } & Me gusta & 9 & 13.85 \\
\hline & No me gusta & 5 & 7.69 \\
\hline \multicolumn{2}{|c|}{$\begin{array}{l}\text { Recurso para la formación como futuro profesional } \\
\text { de la música }\end{array}$} & 13 & 20.00 \\
\hline \multicolumn{2}{|c|}{ Logística y organización } & 10 & 15.38 \\
\hline \multicolumn{2}{|l|}{ Falta de sentido } & 7 & 10.77 \\
\hline \multicolumn{2}{|l|}{ No contesto } & 2 & 3.08 \\
\hline \multicolumn{2}{|l|}{ Perdidos } & 1 & 1.54 \\
\hline
\end{tabular}

Fuente: Tabla creada por el autor con la información derivada de la investigación.

Las posibilidades de mejora se potencian con la opinión de los estudiantes, por lo que se incluyó la pregunta: “¿Qué podría hacer tu maestro para impartir mejor esta materia?”. Las respuestas de los estudiantes fueron bastante halagadoras. Solamente un pequeño porcentaje de los estudiantes (4.9\%) afirmó que el maestro necesitaba preparar y explicar mejor sus clases e, inclusive, un porcentaje similar de estudiantes respondió que el maestro no tenía que hacer algo más para impartir mejor sus clases. Sin embargo, 35.8\% de los estudiantes seleccionó la respuesta: "Hacernos participar más activamente", lo cual refleja una importante área de oportunidad. Esos estudiantes, consciente o inconscientemente, saben que su papel en clase no puede ser pasivo, sino que deben involucrarse en la construcción de su conocimiento. Ese resultado representa una llamada de atención para los maestros que imparten esta materia, quienes, como vimos en la revisión bibliográfica y en el marco teórico, deben limitar la cátedra expositiva y preocuparse por implementar estrategias que promuevan procesos de enseñanza y aprendizaje más colaborativos y, sobre todo, significativos. Además, $19.8 \%$ de los estudiantes seleccionó las opciones de respuesta: "Utilizar mejores recursos didácticos" y "Proporcionar más materiales didácticos". Estos resultados, aunque menos alarmantes en comparación con los anteriores, también representan una llamada de atención importante, pues los recursos didácticos constituyen las herramientas que el profesor implementa para optimizar los procesos de enseñanza y aprendizaje (Tabla 9).

ESCENA. Revista de las artes, 2020, Vol. 80, Núm. 1 (julio-diciembre), pp.148-178 
La Clase de Historia de la Música desde la perspectiva de los estudiantes de la

Artículo

Licenciatura en Música de la Universidad Autónoma de Aguascalientes, México

Tabla 9. Opinión de los estudiantes sobre las mejoras para impartir mejor su materia.

\begin{tabular}{|l|c|c|c|}
\hline \multicolumn{1}{|c|}{ Opción de respuesta } & Frecuencias & Porcentaje & $\begin{array}{c}\text { Porcentaje } \\
\text { de casos }\end{array}$ \\
\hline Hacernos participar más activamente & 29 & $35.8 \%$ & $44.6 \%$ \\
\hline Utilizar mejores recursos didácticos & 16 & $19.8 \%$ & $24.6 \%$ \\
\hline Proporcionar más materiales didácticos & 16 & $19.8 \%$ & $24.6 \%$ \\
\hline Preparar mejor sus clases & 4 & $4.9 \%$ & $6.2 \%$ \\
\hline Explicar mejor sus clases & 4 & $4.9 \%$ & $6.2 \%$ \\
\hline Otro: No necesita hacer más & 4 & $4.9 \%$ & $6.2 \%$ \\
\hline No contesto & 4 & $4.9 \%$ & $6.2 \%$ \\
\hline Perdidos & 2 & $2.5 \%$ & $3.1 \%$ \\
\hline Otro: Hacer menos tediosa la clase & 1 & $1.2 \%$ & $1.5 \%$ \\
\hline $\begin{array}{l}\text { Otro: Poner más énfasis en lo que respecta } \\
\text { a los estilos de las obras }\end{array}$ & 1 & $1.2 \%$ & $1.5 \%$ \\
\hline Total & 81 & $100.0 \%$ & $124.6 \%$ \\
\hline
\end{tabular}

Fuente: Tabla creada por el autor con la información derivada de la investigación

Como al derecho de exigir al maestro corresponde la responsabilidad del estudiante, se incluyó la pregunta: “¿Qué podrías hacer tú, para tener un mejor desempeño en la clase?". Desde la perspectiva del autor, los estudiantes parecen ser bastante sinceros con sus respuestas. Si bien es obligación del maestro planificar y preparar a consciencia los procesos de enseñanza y aprendizaje, este nada puede hacer si los estudiantes no se hacen responsables de sus deberes. Así, $47.6 \%$ de los estudiantes coincidió en que deben leer sus apuntes periódicamente (algo que muchos de ellos suelen dejar para el día anterior al examen), 20.2\% estuvo de acuerdo en interesarse más por la materia y $17.9 \%$ afirmó que debía poner más atención (Tabla 10). 
Tabla 10. Opinión de los estudiantes sobre lo que ellos deberían hacer para tener un mejor desempeño en clase.

\begin{tabular}{|l|c|c|c|}
\hline \multicolumn{1}{|c|}{ Opción de respuesta } & $\begin{array}{c}\text { Número de } \\
\text { Frecuencias }\end{array}$ & Porcentaje & $\begin{array}{c}\text { Porcentaje } \\
\text { de casos }\end{array}$ \\
\hline Leer mis apuntes periódicamente & 40 & $47.6 \%$ & $61.5 \%$ \\
\hline Interesarme más en la materia & 17 & $20.2 \%$ & $26.2 \%$ \\
\hline Poner más atención & 15 & $17.9 \%$ & $23.1 \%$ \\
\hline Cambiar mi opinión respecto a la materia & 5 & $6.0 \%$ & $7.7 \%$ \\
\hline Otro: Ahondar en las temáticas & 2 & $2.4 \%$ & $3.1 \%$ \\
\hline $\begin{array}{l}\text { Otro: Investigar más sobre los temas que } \\
\text { hemos visto en clase }\end{array}$ & 2 & $2.4 \%$ & $3.1 \%$ \\
\hline $\begin{array}{l}\text { Otro: Creo que mi desempeño es muy } \\
\text { adecuado }\end{array}$ & 1 & $1.2 \%$ & $1.5 \%$ \\
\hline $\begin{array}{l}\text { Otro: Buscar textos y documentos para } \\
\text { complementar mi conocimiento }\end{array}$ & 1 & $1.2 \%$ & $1.5 \%$ \\
\hline No contesto & 1 & $1.2 \%$ & $1.5 \%$ \\
\hline
\end{tabular}

Fuente: Tabla creada por el autor con la información derivada de la investigación

Como se sabe, la evaluación es una tarea permanente en cualquier proceso de enseñanza y de aprendizaje. Su propósito principal es conocer qué tanto ha aprendido el estudiante, así como la calidad de lo aprendido, con la finalidad de que esa información sirva para optimizar la labor docente y ofrecer retroalimentación a los estudiantes sobre sus fortalezas y áreas de oportunidad; es decir, lo que le da elementos para mejorar (Stanley, Brooker \& Gillbert, 2002). En ese sentido, uno de los aspectos más delicados de la evaluación está representado por la validez y la confiabilidad. En otras palabras, el estudiante debe sentir que realmente está siendo evaluado en los aspectos abordados en clase y que esa evaluación refleja de manera real, clara y objetiva su nivel de aprendizaje. Por lo anterior, se incluyó el ítem "¿Qué procedimientos de evaluación implementa tu maestro?”, seguido de la instrucción "subraye todos los incisos que sean pertinentes". Las opciones proporcionadas fueron:

a) Examen escrito consistente principalmente en preguntas abiertas a las que hay que responder con un pequeño ensayo.

b) Examen escrito consistente principalmente en preguntas de opción múltiple o paréntesis 
La Clase de Historia de la Música desde la perspectiva de los estudiantes de la

c) Reconocimiento de fragmentos de obras

d) Presentación en clase

e) Trabajo final

f) Presentación de programa de radio "Ventana al Sonido"

g) Otro

Debido a que los estudiantes formaban parte de cuatro grupos bien definidos, fue fácil determinarlos, como se verá en la tabla 11:

Tabla 11. Procedimientos de evaluación implementados en cada clase.

\begin{tabular}{|c|c|c|c|}
\hline $\begin{array}{c}\text { Música y } \\
\begin{array}{c}\text { Sociedad Medieval } \\
\text { y Renacentista }\end{array}\end{array}$ & $\begin{array}{c}\text { Música } \\
\text { y Sociedad } \\
\text { en el Siglo XVIII }\end{array}$ & $\begin{array}{c}\text { Cultura } \\
\text { y Apreciación } \\
\text { Musicales VI }\end{array}$ & $\begin{array}{c}\text { Cultura } \\
\text { y Apreciación } \\
\text { Musicales VIII }\end{array}$ \\
\hline a, c, dyf & b, c, eyf & a yf & d, eyf \\
\hline
\end{tabular}

Fuente: Tabla creada por el autor con la información derivada de la investigación

Figura 3. ¿Qué tan objetivas y justas son las evaluaciones?

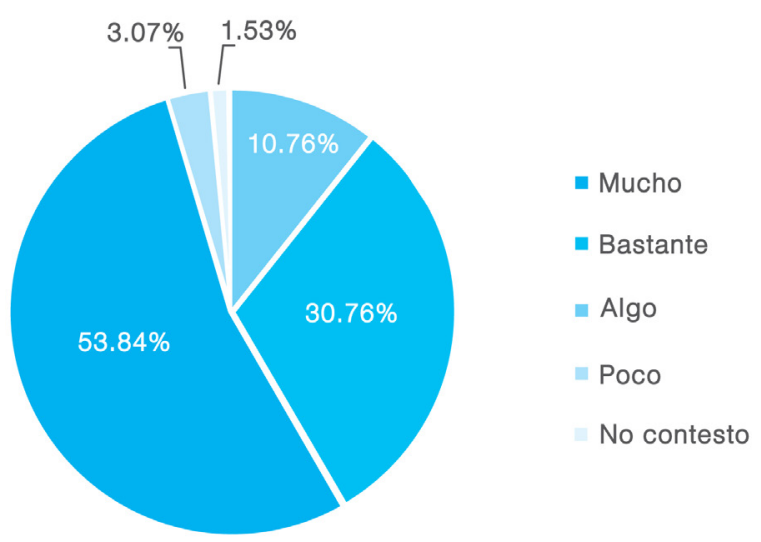

Fuente: Creación del autor con los datos derivados de la investigación.
Como puede verse, dos de los profesores trataron de diversificar los procedimientos de evaluación, mientras que los otros dos parecen preferir un número más reducido de estrategias. Sin embargo, todos los profesores incluyeron la participación en el programa de radio.

Respecto a qué tan objetivas y justas eran las evaluaciones, información que se obtuvo a través del ítem "¿Qué tan objetiva y justa te parece la forma de evaluar de tu maestro?", $53.84 \%$ de los estudiantes afirmaron considerarla muy objetiva y justa. Mientras que $30.76 \%$ declararon que bastante objetiva y justa (Figura 3). 
Con el propósito de confirmar la congruencia del estudiante con las respuestas anteriores, se incluyó el ítem "¿Cómo te gustaría que tu maestro evaluara tu aprendizaje en esta clase?" (Tabla 12). Como la pregunta fue de tipo abierto, se llevó a cabo el análisis descrito y se determinaron tres categorías:

a) Respuestas que reflejan que el estudiante está satisfecho totalmente con el procedimiento de evaluación

b) Respuestas que indican que el estudiante prefiere alguno de los procedimientos ya empleado por el profesor

c) Respuestas que reflejan interés por parte del estudiante para que el profesor emplee algún procedimiento de evaluación alternativo.

Tabla 12. Procedimiento de evaluación que gustaría al estudiante

\begin{tabular}{|l|c|c|}
\hline \multicolumn{1}{|c|}{ Procedimiento de evaluación que gustaría el estudiante } & Frecuencia & Porcentaje \\
\hline No contestó & 7 & $10.77 \%$ \\
\hline Otras alternativas de evaluación & 12 & $18.46 \%$ \\
\hline Alguna de los procedimientos ya empleados por el maestro & 19 & $29.23 \%$ \\
\hline Los mismos procedimientos ya empleados por el maestro & 27 & $41.54 \%$ \\
\hline
\end{tabular}

Fuente: Tabla creada por el autor con la información derivada de la investigación.

Las respuestas incluidas en la primera categoría (41.54\%) parecen confirmar la satisfacción de los estudiantes con los procedimientos de evaluación. Las respuestas incluidas en la segunda categoría (29.23\%) reflejan preferencia hacia alguno de los procedimientos ya empleados. Sin embargo, las respuestas que conformaron la tercera categoría (18.46\%), dan luz sobre el interés de algunos de los estudiantes por hacer más significativa esta dimensión del proceso de enseñanza y aprendizaje, y proponen procedimientos alternativos que parecen haber sido tomados de la revisión bibliográfica de este trabajo. Dentro de las respuestas proporcionadas por los estudiantes, destacaron las siguientes:

- Trabajos en los que se emplee lo visto en clase en vez de un examen. Por ejemplo, sobre documentales, películas históricas, presentaciones, conferencias, entre otros.

- Con la ejecución de alguna obra en clase, según la correcta realización con el estilo de la época.

- Con el estudio de una obra en particular y a partir de esta obtener todos los elementos musicales teóricos y artísticos y filosóficos, entre otros.

- Con pláticas abiertas, como debates, exposiciones y ponencias. 
La Clase de Historia de la Música desde la perspectiva de los estudiantes de la

Artículo

Licenciatura en Música de la Universidad Autónoma de Aguascalientes, México

Como se ha visto hasta aquí, las respuestas a las preguntas anteriores revelaron que, en general, los estudiantes gustan de la clase de Historia de la Música y la perciben como una asignatura útil. No obstante, la pregunta "¿En la actualidad, qué lugar ocupa la clase de historia de la música en tu escala de prioridades?" obtuvo respuestas bastante preocupantes, ya que $35.4 \%$ de los estudiantes afirmó que ocupa el tercer lugar después de su clase de instrumento musical; $18.5 \%$ informó que es la clase menos importante de las materias que toman; $15.4 \%$ respondió que está en primer lugar de importancia al igual que su clase de instrumento; 9.2\% afirmó que ocupa el segundo lugar de importancia, después de su clase de instrumento, y $9.2 \%$ informó que es igual de importante que todas las clases. Finalmente, $7.7 \%$ respondió que ocupa el $4 .^{\circ}$ lugar de importancia y $4.6 \%$ no respondió (Tabla 13). Esta pregunta también incluyó la opción de respuesta "otro".

Tabla 13. Lugar que ocupa la materia en la escala de prioridades del estudiante

\begin{tabular}{|l|c|c|}
\hline \multicolumn{1}{|c|}{ Opción de respuesta } & Frecuencia & Porcentaje \\
\hline $\begin{array}{l}\text { Ocupa el tercer lugar de importancia después que mi clase } \\
\text { de instrumento }\end{array}$ & 23 & $35.4 \%$ \\
\hline Es la clase menos importante de todas las materias que tomo & 12 & $18.5 \%$ \\
\hline Está en primer lugar al igual que mi clase de instrumento & 10 & $15.4 \%$ \\
\hline $\begin{array}{l}\text { Ocupa el segundo lugar de importancia después de mi clase } \\
\text { de instrumento }\end{array}$ & 6 & $9.2 \%$ \\
\hline Es igual de importante que todas las clases & 6 & $9.2 \%$ \\
\hline $\begin{array}{l}\text { Ocupa el 4. }{ }^{\circ} \text { lugar de importancia después que mi clase } \\
\text { de instrumento }\end{array}$ & 5 & $7.7 \%$ \\
\hline No contesto & 3 & $4.6 \%$ \\
\hline
\end{tabular}

Fuente: Tabla creada por el autor con la información derivada de la investigación

\section{Conclusiones}

El estudio llevado a cabo permitió alcanzar los objetivos planteados. En primer lugar, fue alentador determinar el alto grado de identificación de los estudiantes de la Licenciatura en Música con respecto a la asignatura de Historia de la Música. Un resultado contrario habría despertado grandes sospechas sobre el desempeño docente y sobre la calidad, misión y visión humanista de la institución.

Del mismo modo, fue gratificante conocer la percepción que los estudiantes tienen acerca del valor de esta asignatura en su formación profesional y la función práctica que le 
asignan, no solo como recurso para impulsar interpretaciones musicales informadas, sino también como parte indispensable de su cultura profesional general. Igualmente, la información recabada sobre los aspectos que el maestro debería enfatizar arroja luz sobre las expectativas académico-docentes de los estudiantes y representa información valiosa para fortalecer los programas de materia y los planes de clase.

La investigación también permitió caracterizar las acciones y recursos que, desde la perspectiva de los estudiantes, los profesores deberían utilizar para promover un proceso de enseñanza y aprendizaje más eficiente, efectivo y significativo. En ese sentido, la información recabada no puede ser más valiosa. Los estudiantes reclaman una participación más activa y significativa en la construcción de su conocimiento y esa petición es más que auténtica y válida.

La Historia de la Música es una asignatura importante en la formación de los nuevos profesionales de la disciplina; contribuye a su formación general, a su desarrollo académico, intelectual y cultural y, sobre todo, a su proyección artístico-musical. Desgraciadamente, el modelo didáctico-pedagógico que se ha estado siguiendo, no favorece un aprendizaje significativo y promueve una actitud pasiva por parte del estudiante. Es imperativo que el maestro reduzca la cátedra expositiva e incremente diversas estrategias para impulsar el involucramiento activo de los estudiantes. Como afirman Carvalho-Azevedo y Ferreira-Correa, "ampliar las formas de interacción del alumnado/aprendiz con su objeto de estudio puede promover y desarrollar la comprensión de ese objeto" (2014, p. 6). Por supuesto, no se trata de cambiar totalmente los modelos establecidos, sino de renovarlos, de volverlos vivenciales, de hacerlos significativos. No en vano Bush enfatiza: "Encontrar actividades interesantes y apropiadas para dar vida a una materia es siempre un gran reto" (2000, p. 31). Como docente, cada quien debería preguntarse si quizá por esa razón, la clase de Historia de la Música no ocupa el mismo lugar de importancia que las demás asignaturas, como indicaron en su mayoría los resultados de este estudio.

La investigación indica que los estudiantes están conscientes de las acciones que deben emprender para que los procesos de enseñanza y aprendizaje sean más eficientes, efectivos y significativos. De hecho, un porcentaje considerable de ellos aceptó que debía leer sus apuntes periódicamente, además, muchos estuvieron de acuerdo en que debían interesarse más por la materia.

Finalmente, aun cuando las respuestas de los estudiantes reflejan satisfacción respecto a los mecanismos de evaluación, las respuestas también reflejaron un alto nivel de madurez y un genuino interés por la diversificación en los medios de evaluación. Además, presentan bastante claridad sobre cómo lograrlo, al proponer que la evaluación se llevara a cabo a través de trabajos sobre documentales, películas históricas, presentaciones o 
La Clase de Historia de la Música desde la perspectiva de los estudiantes de la

Artículo

Licenciatura en Música de la Universidad Autónoma de Aguascalientes, México

conferencias; con la ejecución de alguna obra en clase en estricto seguimiento al estilo de la época; con el estudio de una obra específica, a partir de la cual se abordaran aspectos teóricos, históricos, artísticos y filosóficos o, mejor aún, a través de pláticas abiertas, como debates, exposiciones y presentaciones de ponencias.

Así, el autor propone un cambio radical en la impartición de esa asignatura que incluya, por un lado, un enfoque musicológico interdisciplinar, que lo mismo involucre la sociología que la antropología; la filosofía que la acústica. Por el otro, una participación vivencial por parte de los estudiantes en su propia formación. También se requiere de un cambio de raíz en los criterios que muchas veces se han establecido para la selección del profesorado que la imparte. Es casi del dominio público que muchos maestros de instrumento dedican parte de su tiempo laboral a la impartición de esta materia para poder llenar su carga académica, lo que ocasiona que muchas veces sea impartida de una manera superficial. Si se quiere mejorar los procesos de enseñanza y aprendizaje de la Historia de la Música, es necesario seleccionar docentes debidamente habilitados para impartirla.

\section{Referencias}

Ausubel, D. P. (1976). Psicología educativa. Un punto de vista cognoscitivo. Ed. Trillas. México.

Ausubel, P. D. (2000). Adquisición y Retención del Conocimiento. Una Perspectiva Cognitiva. España: Ediciones Paidós Ibérica.

Burkholder, J. P. (2011). Decoding the Discipline of Music History for Our Students. Journal of Music History Pedagogy, 1(2), 93-111.

Bush, J. E. (2000). Bringing Music History to Life. Music Educators Journal, 5, 31-52.

Carvalho-Azevedo M. C. \& Ferreira-Correa, A. (2014). Sobre la función de la asignatura de Historia de la Música en la formación del profesorado de música. Revista Internacional De Educación Musical, 2, 2-9. https://doi.org/10.12967/riem-20142-p002-009

Casas, M.V., Escobar, M \& Burbano, R. M. (2014). Miradas desde la Historia de la Música a la formación profesional de músicos en Colombia: resultados preliminares. Historelo Revista de Historia Regional y Local, 6(12), 376-412.

Cochran, T. B. (2019). Reading Primary Sources Analytically. Journal of Music History Pedagogy, 9(1), 79-89.

Conor, E. (2019). Re-envisioning Information Literacy: Critical Information Literacy, Disciplinary Discourses, and Music History. Journal of Music History Pedagogy, 9(1), 28-43.

ESCENA. Revista de las artes, 2020, Vol. 80, Núm. 1 (julio-diciembre), pp. 148-178 
Cypess, R. (2019). Historical Thinking and Individual Creativity: Teaching Primary Sources on Performance. Journal of Music History Pedagogy, 9(1), 90-98.

Davis, J. A. (2010). Classroom Discussion and the Community of Music Majors. Journal of Music History Pedagogy, 1(1), 5-17.

De Luca, M. R. (2016). Constructing Music History in the Classroom. Musica Docta. Rivista digitale di Pedagogia e Didattica della musica, 6(1), 113-121.

Dirkse, S. (2011). Encouraging Empirical Research: Findings from the Music Appreciation Classroom. Journal of Music History Pedagogy, 2(1), 25-35.

Escobar-Pérez, J. \& Cuervo-Martínez, Á. (2008). Validez de contenido y juicio de expertos: una aproximación a su utilización. Avances en Medición, 6, 27-36.

Haefeli, S. (2013). Using Blogs for Better Student Writing Outcomes. Journal of Music History Pedagogy, 4(1), 39-70.

Kincheloe, J. L. \& McLaren, P. (2005). Rethinking critical theory and qualitative research. En N. K. Denzin y Y. S. Lincoln (Eds.). The Sage Handbook of Qualitative Research. Thousand Oaks: Sage.

Kraemer, R. D. (2000). Dimensoes e funcoes do conhecimiento pedagógico musical. Em Pauta, 11(16), 48-73.

Knyt, E. E. (2013). Rethinking the Music History Research Paper Assignment. Journal of Music History Pedagogy, 4(1), 23-37.

Kuykendall, J. B. (2019). Armchair Philology in the Post-Truth Age. Journal of Music History Pedagogy, 9(1), 99-112.

López-Cano, R. (2007). Musicología vs. Etnomusicología ¿Un falso debate? Etno-Boletín Informativo de La SIbE, 16, 6-10.

Lowe, M. (2010). Teaching Music History Today: Making Tangible Connections to Here and Now. Journal of Music History Pedagogy, 1(1), 28-43.

Montoya-Rubio, J. C. (2016). La musicología como ancla de la educación musical: del historicismo al audiovisual. La Albolafia: Revista de Humanidades y Cultura, 9, 6173.

Morales-Ortiz, B. L. (2012). Historia de la Música en los Programas de Licenciatura en Música. El Artista, 9, 312-335.

Morgan-Ellis, E. (2019). A Faculty Learning Community. Journal of Music History Pedagogy, 9(2), 173-193 .

ESCENA. Revista de las artes, 2020, Vol. 80, Núm. 1 (julio-diciembre), pp.148-178 
La Clase de Historia de la Música desde la perspectiva de los estudiantes de la Artículo Licenciatura en Música de la Universidad Autónoma de Aguascalientes, México

Nettl, B. (1995). Heartland Excursions: Ethnomusicological Reflections on Schools of Music. Champaign: University of Illinois Press.

Solorio-Farfán, H. \& Capistrán-Gracia, R.W. (2019). Nadar a contracorriente: El tratamiento histórico del objeto desde la musicología. En R. W. Capistrán Gracia (Coord.), Abordajes Inter, Multi y Transdisciplinares en Torno al Arte y la Cultura (pp. 127146). Aguascalientes, México: Editorial Universidad Autónoma de Aguascalientes.

Stake, R. E. (2005). Investigación con estudios de caso. Madrid: Morata.

Stanley, M., Brooker, R. \& Gilbert, R. (2002). Examiner perceptions of using criteria in music performance assessment. Research Studies in Music Education, 18(1), 4656. doi:10.1177/1321103X020180010601.

Stimeling, T. D \& Katz, M. (2012). Songwriting as Musicological Inquiry: Examples from the Popular Music Classroom. Journal of Music History Pedagogy, 2(2), 133-152.

Yang, S. (2012). Singing Gesualdo: Rules of Engagement in the Music History Classroom. Journal of Music History Pedagogy, 3(1), 39-55. 